A C T A C H E M I A S C A N D I N A V I C A 17 (1963) S 195-S 202

\title{
Equilibrium Reaction Rates and Enzyme Mechanisms*
}

\author{
P. D. BOYE R** and E. S I LVERSTEI N*** \\ Department of Biochemistry, University of Minnesota Medical School, \\ Minneapolis 14, Minnesota, USA
}

\begin{abstract}
General relationships are developed for rates of substrate interchange at equilibrium for enzyme reactions of the type $A+B \rightleftharpoons C+D$ and $\mathrm{A}+\mathrm{B} \rightleftharpoons \mathrm{C}$ proceeding through interconversion of ternary complexes and with compulsory or with alternative orders of addition of substrates. Usefulness of such measurements for determination of substrate binding order and of ternary complex interconversion as a possible rate-limiting step, for setting minimum values for dissociation constants, and for detection of other aspects of catalytic mechanisms are presented and discussed.
\end{abstract}

M

any approaches are essential for study of enzyme mechanisms. For example, measurements of initial reaction velocities and of substrate and inhibitor binding under various conditions have been powerful tools in the skilful hands of Professor Theorell and his associates, and it is with pleasure that this paper is dedicated to Professor Theorell. A new approach which may deserve considerably more attention is the measurement of reaction rates at equilibrium, as made possible by isotopic techniques. Biochemists have made frequent and valuable use of measurements of exchange reactions, but without consideration of the fundamental relationships governing such exchange rates in enzyme systems. Rate measurements at equilibrium, although technically somewhat difficult, have the theoretical advantage that all reaction intermediates are at equilibrium concentration, thus considerably simplifying derivation and interpretation.

Some theoretical bases for equilibrium reaction rate studies in enzyme systems were first presented by Boyer in $1959^{1}$, and have been correlated with experimental observations in subsequent studies from his laboratory ${ }^{2-4}$. The reports have stimulated a generalized treatment by Morales and co-workers ${ }^{5}$ of transient states of tracer distribution in enzyme systems where the amount of labeled substrate combined with enzyme may be appreciable, and of considerations by Alberty et al..$^{6}$ of alternative means of deriving the appropriate relationships for simplified linear sequences.

* Supported in part by grants from the U. S. Public Health Service and the Hill Family Foundation.

** Present address: Department of Chemistry, University of California, Los Angeles 24, California.

*** American Cancer Society Post-Doctoral Fellow. 
Previous considerations have not included enzyme reactions proceeding through interconversion of two ternary complexes and with either alternative or compulsory order of addition of substrates. The purpose of this paper is to present some appropriate equations and their implications. These serve as the basis for studies of equilibrium reaction rates with liver and yeast alcohol dehydrogenase, with heart and skeletal muscle lactate dehydrogenases, and with hexokinase which have quite recently been completed in our laboratory ${ }^{4}$.

\section{DERIVATION OF BASIC RELATIONSHIPS}

The approach previously used ${ }^{1}$ leads readily to general equations governing rates of interchange of reactants in enzyme systems as described by eqn. (1).*

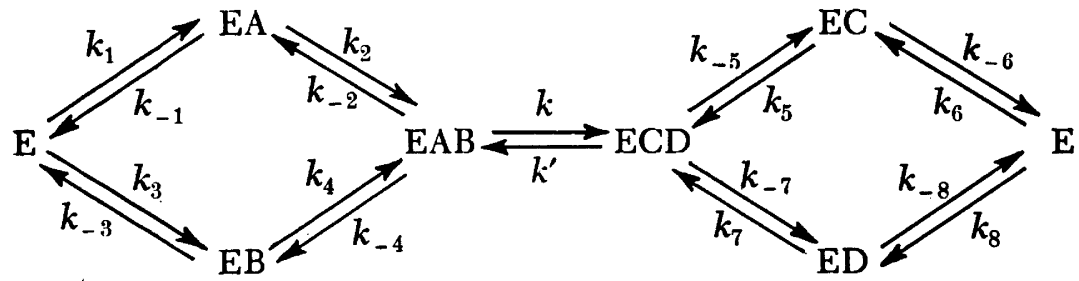

For example, for the rate, $\mathrm{R}$, of interconversion of $\mathrm{A}$ with $\mathrm{C}$ at equilibrium, let

$$
\begin{aligned}
& \mathbf{E A}=p+q \text {, where } p=\mathbf{E A} \text { derived most recently from } \mathbf{A}^{* *} \\
& \text { and } q=" \quad \text { " } \quad \text { " } \quad \text { " C; } \\
& \mathrm{EAB}=w+x \text {, where } w=\mathrm{EAB} \quad " \quad " \quad, \quad " \quad \mathrm{~A} \\
& \text { and } x=" \quad " \quad, \quad " \quad \text { " } \quad \text { C; } \\
& \mathrm{ECD}=y+z \text {, where } y=\mathrm{ECD} \quad " \quad \quad \quad \quad \quad " \quad \mathbf{A} \\
& \text { and } z=" \quad " \quad, \quad " \quad, \quad \text { " C; } \\
& \mathrm{EC}=m+n \text {, where } m=\mathrm{EC} \quad \text { " } \quad \text { " } \quad \text { " } \mathrm{A} \\
& \text { and } n=" \quad, \quad, \quad \quad " \quad, \quad \text { C. }
\end{aligned}
$$

The enzyme conservation equation and the various equilibria involved lead to

$$
\mathrm{E}_{t}=(y+z)\left[1+\frac{k}{k^{\prime}}\left(1+\frac{K_{2}}{\mathrm{~B}}+\frac{K_{4}}{\mathrm{~A}}+\frac{K_{1} K_{2}}{\mathrm{AB}}\right)+\frac{K_{5}}{\mathrm{D}}+\frac{K_{7}}{\mathrm{C}}\right]
$$

where $K_{1}, K_{2}$, etc. are the respective dissociation constants and $\mathrm{A}, \mathrm{B}$, etc. refer to reactant concentrations. In the system given by eqn. (1), $\mathrm{R}=k_{-7} y+$ $k_{-6} m$, and from this and the relation $\mathrm{d} m / \mathrm{d} t=\mathrm{O}=k_{-5} y-k_{5} m \mathrm{D}-k_{-6} m$, one obtains $y=\mathrm{R}\left(k_{-6}+k_{5} \mathrm{D}\right) /\left(k_{-5} k_{-6}+k_{-6} k_{-7}+k_{5} k_{-7} \mathrm{D}\right)$. Similarly, $\mathrm{R}$ also $=k_{-4} x$ $+k_{-1} q$, and from this and the relation $\mathrm{d} q / \mathrm{d} t=\mathrm{O}=k_{-2} x-k_{2} q \mathrm{~B}-k_{-1} q$ one obtains

* Relationships for rates of isotopic interchange in linear systems or systems where only one ternary complex is formed, whether at equilibrium or considerably displaced from equilibrium, may be obtained from initial velocity equations as developed by Cleland ${ }^{7}$ (W. W. Cleland, personal communication). The initial velocity equation for a scheme such as given by eqn. (1) is extremely complex, and the simple derivation given herein is preferable for the equilibrium situation.

** The chemical properties of the various enzyme-substrate combinations are obviously independent of their route of formation. The useful concept given here can also be visualized in terms of specific activities. For example, if isotope is added as $\mathbf{A}$ and negligible isotope is present in $\mathrm{C}$, the ratio $p / q \times$ specific activity of $\mathrm{A}=$ specific activity of $\mathrm{EA}$. 
$x=\mathrm{R}\left(k_{-1}+k_{2} \mathrm{~B}\right) /\left(k_{-1} k_{-2}+k_{-1} k_{-4}+k_{2} k_{-4} \mathrm{~B}\right)$. In addition, $\mathrm{R}=k^{\prime} z-k x$, which allows solving for $z$ in terms of $x$ and $\mathrm{R}$. Substitution of the values for $z$ and $y$ into eqn. (2) leads to general eqn. (3).*

$$
\begin{aligned}
\mathrm{R}=\mathrm{E}_{t} /\{[ & \left.1+\frac{k^{\prime}\left(k_{-6}+k_{5} \mathrm{D}\right)}{k_{-5} k_{-6}+k_{-7}\left(k_{-6}+k_{5} \mathrm{D}\right)}+\frac{k\left(k_{-1}+k_{2} \mathrm{~B}\right)}{k_{-1} k_{-2}+k_{-4}\left(k_{-1}+k_{2} \mathrm{~B}\right)}\right] \times \\
& \left.\times\left[\frac{1}{k^{\prime}}\left(1+\frac{K_{5}}{\mathrm{D}}+\frac{K_{7}}{\mathrm{C}}\right)+\frac{1}{k}\left(1+\frac{K_{2}}{\mathrm{~B}}+\frac{K_{4}}{\mathrm{~A}}+\frac{K_{1} K_{2}}{\mathrm{AB}}\right)\right]\right\}
\end{aligned}
$$

The rate of $\mathrm{B} \rightleftharpoons \mathrm{D}$ interconversion, $\mathrm{R}^{\prime}$, is given by a similar expression as follows:

$$
\begin{gathered}
\mathrm{R}^{\prime}=\mathrm{E}_{t} /\left\{\left[1+\frac{k^{\prime}\left(k_{-8}+k_{7} \mathrm{C}\right)}{k_{-7} k_{-8}+k_{-5}\left(k_{-8}+k_{7} \mathrm{C}\right)}+\frac{k\left(k_{-3}+k_{4} \mathrm{~A}\right)}{k_{-3} k_{-4}+k_{-2}\left(k_{-3}+k_{4} \mathrm{~A}\right)}\right] \times\right. \\
\left.\times\left[\frac{1}{k^{\prime}}\left(1+\frac{K_{5}}{\mathrm{D}}+\frac{K_{7}}{\mathrm{C}}\right)+\frac{1}{k}\left(1+\frac{K_{2}}{\mathrm{~B}}+\frac{K_{4}}{\mathrm{~A}}+\frac{K_{1} K_{2}}{\mathrm{AB}}\right)\right]\right\}
\end{gathered}
$$

Relationships for compulsory pathways (linear sequences) are readily derived, as limiting cases of general relationships, such as eqns. (3) and (4). For example, for the pathway involving only the upper part of eqn. (1), the terms $K_{4}, K_{7}, k_{-4}$ and $k_{-7}$ are set equal to 0 to give:

$$
\begin{gathered}
\mathrm{R}=\mathrm{E}_{t} /\left\{\left[1+\frac{k^{\prime}\left(k_{-6}+k_{5} \mathrm{D}\right)}{k_{-5} k_{-6}}+\frac{k\left(k_{-1}+k_{2} \mathrm{~B}\right)}{k_{-1} k_{-2}}\right] \times\right. \\
\left.\times\left[\frac{1}{k^{\prime}}\left(1+\frac{K_{5}}{\mathrm{D}}\right)+\frac{1}{k}\left(1+\frac{K_{2}}{\mathrm{~B}}+\frac{K_{1} K_{2}}{\mathrm{AB}}\right)\right]\right\} \\
\mathrm{R}^{\prime}=\mathrm{E}_{t} /\left[1+\frac{k^{\prime}}{k_{-5}}+\frac{k}{k_{-2}}\right]\left[\frac{1}{k^{\prime}}\left(1+\frac{K_{5}}{\mathrm{D}}\right)+\frac{1}{k}\left(1+\frac{K_{2}}{\mathrm{~B}}+\frac{K_{1} K_{2}}{\mathrm{AB}}\right)\right]
\end{gathered}
$$

Unwarranted confusion and apparent discrepancy may arise because of lack of clarity in definition rather than experimental result. For reaction proceeding through complexes between an enzyme and two or more substrates, the following definitions apply to terms as used in this paper and are suggested for general usage:

1. Random order or sequence of substrate addition. The addition of substrates to an enzyme or their dissociation from enzyme-substrate complexes occurs at equal rate whether or not another substrate or other substrates are bound to the enzyme. In terms of eqn. (1), this means that $k_{1}=k_{4}, k_{-1}=k_{-4}, k_{2}=k_{3}$, etc.

2. Alternative order or sequence of substrate addition. Complexes of enzyme with more than one substrate may be formed by prior addition of any of the substrates, and similarly, dissociation of substrates may occur in any order. Alternative order of addition thus includes the random pathway, but also includes enzymes for which the rate constant for binding of a substrate to the enzyme is greater or less than the rate constant for binding when another substrate is already present on the enzyme. Alternative orders with differences in rate constants

* The equalities $K_{1} K_{2} / k \mathrm{AB}=K_{3} K_{4} / k \mathrm{AB}=K_{5} K_{6} / k^{\prime} \mathrm{CD}=K_{7} K_{8} / k^{\prime} \mathrm{CD}$ obviously obtain, and these terms may be used interchangeably in the denominator.

For many purposes, the form of eqn. (3) as given here is preferable to the form of equations given previously for simpler systems. For example, eqn. (45) of Boyer ${ }^{1}$ may be written:

$$
\begin{aligned}
\mathrm{R}=\mathrm{E}_{t} /\left\{\left[\frac{k_{-8}+k_{7} \mathrm{D}}{k_{-5} k_{-8}+k_{-7}\left(k_{-8}+k_{7} \mathrm{D}\right)}+\right.\right. & \left.\frac{k_{-1}+k_{2} \mathrm{~B}}{k_{-1} k_{-2}+k_{-4}\left(k_{-1}+k_{2} \mathrm{~B}\right)}\right] \times \\
& \left.\times\left[1+\frac{K_{4}}{\mathrm{~A}}+\frac{K_{2}}{\mathrm{~B}}+\frac{K_{1} K_{2}}{\mathrm{AB}}+\frac{K_{5}}{\mathrm{C}}+\frac{K_{7}}{\mathrm{D}}\right]\right\}
\end{aligned}
$$

Acta Chem. Scand. 17 (1963) Suppl. 1 
for binding must also have differences in some rate constants for dissociation for equilibrium to be maintained.

The designation branched sequence is synonymous with alternative order of substrate addition, and, similarly, includes but is not limited to random addition.

Designations such as "partially compulsory" or "partially random" may be appropriate for patterns of behavior of certain enzymes with branched sequences.

3. Compulsory order or sequence substrate binding. The addition of one specific substrate must occur before another substrate can be bound, and similarly, dissociation of a certain substrate must occur before another substrate can dissociate.

The designations linear, ordered, or obligatory sequences are synonymous with a compulsory sequence.

Mechanisms of appropriate enzymes are best described in relation to possible orders of substrate addition as defined above, and not in relation to the order that may exist under prescribed conditions of substrate concentration, $\mathrm{pH}$, etc. Thus an enzyme with a branched mechanism may, under appropriate conditions, catalyze net reaction almost entirely through ordered addition of substrates. This may be appropriately described as kinetically ordered under prescribed conditions, but the enzyme should not be described as having a branched or ordered mechanism.

When one pair of substrates is at high concentration, simplified relationships are also obtaiend. Thus if $B$ and $D$ are increased until they no longer influence $R$, general eqn. (3) for the alternative pathway becomes

$$
\mathrm{R}=\mathrm{E}_{t} /\left[1+\frac{k^{\prime}}{k_{-7}}+\frac{k}{k_{-4}}\right]\left[\frac{1}{k^{\prime}}\left(1+\frac{K_{7}}{C}\right)+\frac{1}{k}\left(1+\frac{K_{4}}{\mathrm{~A}}\right)\right]
$$

When all reactants are at high concentration, eqn. (3) becomes

$$
\mathrm{R}=\mathrm{E}_{t} /\left(1+\frac{k^{\prime}}{k_{-7}}+\frac{k}{k_{-4}}\right)\left(\frac{1}{k^{\prime}}+\frac{1}{k}\right)
$$

and eqn. (4) becomes $\mathrm{R}^{\prime}=\mathrm{E}_{t} /\left(1+\frac{k^{\prime}}{k_{-5}}+\frac{k}{k_{-2}}\right)\left(\frac{1}{k^{\prime}}+\frac{1}{k}\right)$

Relationships for three component systems, such as $\mathrm{A}+\mathrm{B} \rightleftharpoons \mathrm{C}$, are readily obtained from eqns. (3) or (4) by dropping appropriate terms. For example, if $k k_{-5}$

eqn. (1) is modified so that $\mathrm{EAB} \stackrel{\mathrm{EC}}{\rightleftharpoons} \rightleftharpoons \mathrm{E}+\mathrm{C}$, the eqn. (3) terms $k_{-6}, k_{-7}$, $k^{\prime} \quad k_{5}$

$k_{-8}, K_{7} / \mathrm{C}, k_{5} \mathrm{D}, K_{1} K_{2} / k \mathrm{AB}\left(=K_{7} K_{8} / k^{\prime} \mathrm{CD}\right)$ would be dropped with $K_{5} / \mathrm{C}$ replacing $K_{5} / \mathrm{D}$ to give eqn. (10) for $\mathrm{A} \rightleftharpoons \mathrm{C}$.

$\mathrm{R}=\mathrm{E}_{t} /\left[1+\frac{k^{\prime}}{k_{-5}}+\frac{k\left(k_{-1}+k_{2} \mathrm{~B}\right)}{k_{-1} k_{-2}+k_{-4}\left(k_{-1}+k_{2} \mathrm{~B}\right)}\right]\left[\frac{1}{k^{\prime}}\left(1+\frac{K_{5}}{\mathrm{C}}\right)+\frac{1}{k}\left(1+\frac{K_{4}}{\mathrm{~A}}+\frac{K_{2}}{\mathrm{~B}}\right)\right]$

\section{APPLICATIONS OF RELATIONSHIPS}

Compulsory pathways - If a compulsory pathway exists such that either the top or the bottom of eqn. (1) applies, increase in concentration of all reactants or of one or both of the last reactants to add in formation of the ternary complexes will give a predictable and distinguishable rate behavior. For example, as shown by eqn. (5), increase in $\mathrm{B}$ and $\mathrm{D}$ while maintaining equilibrium will result first in an increase, then in progressive inhibition of the $A \rightleftharpoons C$ interchange until complete inhibition occurs; in contrast, the $\mathrm{B} \rightleftharpoons \mathrm{D}$ interchange will increase 
Fig. 1. Theoretical exchange at equilibrium showing decrease in the rate $\mathrm{A} \rightleftharpoons \mathrm{C}$ when dissociation is limited to ternary complexes. The curves are calculated from eqns. (3) and (4) assuming that $\mathrm{E}_{t}=100$, all $K^{\prime} \mathrm{s} k^{\prime}, k$, $k_{2}, k_{-2}, k_{5}$ and $k_{-5}=1 ; k_{4}, k_{-4}, k_{7}$, $k_{-7}=0.1$; and $\left.k_{1}, k_{-1}, k_{6}=2 ; \mathrm{A}\right\rangle \backslash K_{4}$, $\left.\left.\mathrm{B})\rangle K_{2}, k_{4} \mathrm{~A}\right\rangle\right\rangle k_{-3}, k_{-7} k_{-8}$; and $k_{7} \mathrm{C}$ ) $k_{-8}, k_{-3} k_{-4}$.

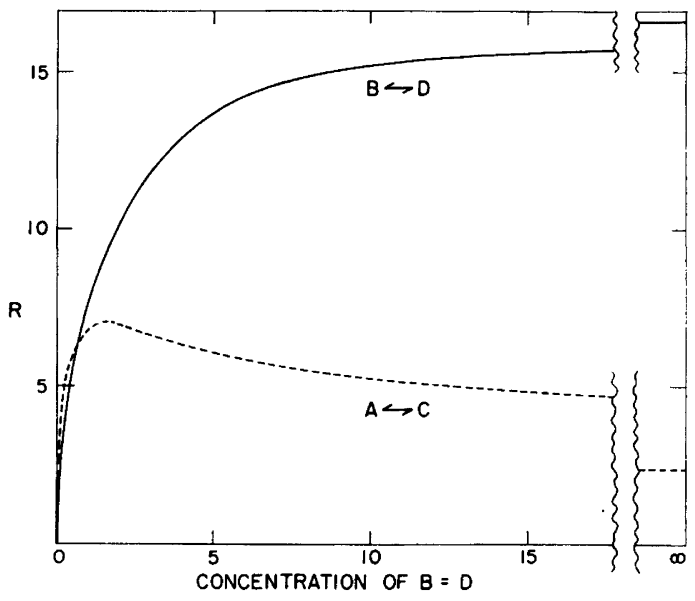

to a maximum. Appropriate experiments will thus readily identify the order of reactant combination in any linear sequence.

If partially compulsory pathways occur, these may be revealed in some circumstances by incomplete inhibition in the interchange rates of one substrate pair as the concentrations of another substrate pair is increased. As B and D are increased from 0 to $\infty$ the left-bracketed term of the denominator of eqn. (3) increases from $1 /\left(k_{-2}+k_{-4}\right)$ to $1 / k_{-4}$, thus tending to decrease $\mathrm{R}$. Conversely, the right-bracketed term decreases, thus tending to increase in $R$. When certain relationships between rate constants hold, these effects may occur relatively independently of one another. Such a possibility is shown in Fig. 1. The conditions for this plot are such that the rate of dissociation of $\mathrm{A}$ from EAB is less than the rate from EA, and that of C from EAC less than that from EC. Thus at high concentration of $B$ and $D$, the interchange $A \rightleftharpoons C$ is effectively limited to the slower dissociation steps.

Results obtained with glutamine synthetase ${ }^{3}$, in which a "hump" in rate plats at equilibrium was observed, may perhaps be ascribed to relationships such as those discussed above.

Ternary complex interconversion as a rate-limiting step. When the rate of ternary complex interconversion, governed by $k$ and $k^{\prime}$ is very small compared to the rate of dissociation of substrates from the ternary complexes, equality of $A \rightleftharpoons C$ and $B \rightleftharpoons D$ interchanges will result at all levels of $A, B, C$, and $D$ for the alternative addition sequence. This may be illustrated, for example, by further examination of eqns. (3) and (4). If $k^{\prime}\left\langle\left\langle k_{-7}\right.\right.$ and $k\left\langle<k_{-4}\right.$, the leftbracketed denominator term of eqn. (3) becomes equal to 1 . A similar situation holds for eqn. (4) if $k^{\prime}\left\langle\left\langle k_{-5}\right.\right.$ and $k\left\langle\left\langle k_{-2}\right.\right.$. When these limitations on rate constants apply, eqn. (3) becomes identical to (4). These limitations are the requirements for the ternary complex interconversion as the rate-limiting step. Thus for the alternative addition of substrates, when $k^{\prime}\left\langle\left\langle k_{-5}, k_{-7}\right.\right.$ and $k\left\langle\left\langle k_{-2}, k_{-4}\right.\right.$, equality of $\mathrm{A} \rightleftharpoons \mathrm{B}$ and $\mathrm{C} \rightleftharpoons \mathrm{D}$ will be observed.

Alberty et al. ${ }^{6}$ have regarded previous deductions ${ }^{1,2,8}$ that equilibrium exchange rates may provide information about rate-limiting steps as being without foundation. In their develop- 
ment they consider only linear sequences, and incorrectly infer that the previous considerations of Boyer et al. ${ }^{1,2,8}$ were similarly limited to linear or compulsory sequences. As noted in this section, and in agreement with earlier deductions ${ }^{1,2,8}$, measurements of exchange rates can give information about rate-limiting steps in branched sequences.

Even with linear sequences inferences about rate-limiting steps may be derived from exchange rate measurements. For example, if ternary complex interconversion was extremely slow, equality of exchanges in a linear mechanism would occur until inordinately high concentrations of the last substrates to add were reached. Also, irrespective of the rate of ternarycomplex interconversion, if the first pair of substrates to add equilibrate very rapidly between free and bound forms, equality of exchanges would be observed until sufficiently high concentrations of the last substrates to add were reached so as to reform the ternary complexes before rapid equilibration with the first substrates could ensue. Thus eqns. (5) and (6) for $R$ and $\mathrm{R}^{\prime}$, compulsory pathways, are identical as long as $\left.k_{-1}\right\rangle>k_{2} \mathrm{~B}$ and $\left.k_{-6}\right\rangle>k_{5} \mathrm{D}$. Further, if exchanges such as those of oxygen among substrates of glutamine synthetase are observed ${ }^{3}$, additional inferences are possible about rate-limiting steps for linear as well as for branched sequences.

Equality of the interchanges $\mathrm{A} \rightleftharpoons \mathrm{B}$ and $\mathrm{C} \rightleftharpoons \mathrm{D}$ might also be observed even when $k^{\prime} \nless<k_{-5}, k_{-7}$ and $k \nless<k_{-2}, k_{-4}$ if a fortuitious equality existed in the leftbracketed denominator terms of eqns. (3) and (4) at certain substrate concentrations, or with excess substrates present and if $k_{-2}=k_{-4}$ and $k_{-7}=k_{-5}$. Such situations would, however, give inequality of interchanges with variations of $B$ and $D$ or $A$ and $C$ at less than saturation levels and thus could readily be detected.

Equalities of reaction rates at low substrate concentrations. When the reaction proceeds by a compulsory sequence and substrate concentrations are high, inequality of $\mathrm{A} \rightleftharpoons \mathrm{C}$ and $\mathrm{B} \rightleftharpoons \mathrm{D}$ must be observed, as noted before, even if the ternary complex interconversion is slow. However, irrespective of the rate of ternary complex interconversion, equalities of exchange will appear as substrate concentrations approach low levels for both linear and branched sequences. For linear sequences, eqns. (5) and (6) become identical when D and B are decreased sufficiently so that $k_{5} \mathrm{D}\left\langle\left\langle k_{-6}\right.\right.$ and $k_{2} \mathrm{~B}\left\langle\left\langle k_{-1}\right.\right.$. Thus the concentration range at which exchange rates in a compulsory sequence approach equality, as $B$ and $D$ are varied and lowered, gives information about the relative magnitudes of $k_{2}$ and $k_{-1}$, and of $k_{5}$ and $k_{-6}$.

With branched sequences, eqns. (3) and (4) become identical when $k_{5} \mathrm{D}\left\langle\left\langle k_{-6}\right.\right.$, $k_{2} \mathrm{~B}\left\langle\left\langle k_{-1}, k_{7} \mathrm{C}\left\langle\left\langle k_{-8}\right.\right.\right.\right.$, and $k_{4} \mathrm{~A}\left\langle\left\langle k_{-3}\right.\right.$. Conditions for equality of exchange with decrease in substrate concentration for branched sequences are thus somewhat more stringent than those for linear sequences.

Relationships to dissociation constants. From eqn. (7) for $\mathrm{A} \rightleftharpoons \mathrm{C}$, random pathway, at high $\mathrm{B}$ and $\mathrm{D}$ concentrations, $\mathrm{R}$ will be a function of $K_{7} / \mathrm{C}$ or $K_{4} / \mathrm{A}$ or both. Under some circumstances it may be possible to adjust equilibria concentrations so that $B, D$, and $C$ remain high so that eqn. (7) becomes of the form

$$
\mathrm{R}=\frac{\mathrm{a} \text { constant }}{1+\frac{k}{k^{\prime}}+\frac{K_{4}}{A}}
$$

A limitation in obtaining eqn. (11) is that B must be sufficiently high so that not only is $K_{2} / \mathrm{B}$ negligible, but $K_{1} K_{2} / \mathrm{AB}$ must remain negligible throughout the concentration range studied. If eqn. (11) applies, the ratio of the slope to intercept of the $1 / \mathrm{R} v s$. 1/A plot will give $K_{4} /\left(1+k / k^{\prime}\right)$, or a minimum value for $K_{4}$. 
Similarly, if A can be maintained high, a minimum value for $K_{7}$ may be obtained. Values obtained from such approaches will give $K_{2}$ and $K_{4}$ each divided by $\left(1+k / k^{\prime}\right)$, but $K_{5}$ and $K_{7}$ each divided by $\left(1+k^{\prime} / k\right)$. If $k=k^{\prime}$, the ratios of slopes to intercepts will give $\frac{12}{2}$ the values of dissociation constants. If $\left.\left.k\right\rangle\right\rangle k^{\prime}$, actual values for $K_{5}$ and $K_{7}$ but far too small values for $K_{2}$ and $K_{4}$ will be obtained, or vice versa if $\left.\left.k^{\prime}\right\rangle\right\rangle k$. Equality of $k$ and $k^{\prime}$ would be unusual fortuity, and thus actual values for dissociation constants of some substrates are likely to be approached. If values of dissociation constants are known from independent studies, the equilibrium rate studies may allow evaluation of the ratio $k / k^{\prime}$.

Similar approaches are applicable to linear sequences, but with dissociation constants given by the concentration dependency of $\mathrm{R}^{\prime}$. For example, when concentrations of $A, B$, and $C$ are held high, the equilibrium rate dependencies will give a minimal value for the dissociation constant of $D$.

Effects of abortive ternary complex formation. The formation of abortive ternary complexes, or of combinations of enzyme with more than one substrate molecule might have definite effects on the rate relationships. For example, the addition of reactions for formation of the complexes EAC and EBD to eqn. (1) leads to relationships like eqns. (3) and (4) but with additional terms involving ratios of $A / C$ and of $B / D$ together with appropriate dissociation constants in the second bracketed term of the denominator. Thus if the ratios of $A / C$ and $B / D$ are held constant, the general form of the equation will not vary. But if, for example, concentrations of $A$ and $D$ are increased, the term involving $B / D$ will drop out but that involving $\mathrm{A} / \mathrm{C}$ will continue to increase with continued fall in $\mathrm{R}$.

Relationships between the chemical transformation rate and equilibrium reaction rate. The rate of interconversion of the ternary complexes, or the actual chemical transformation rate, $\mathrm{R}^{\prime \prime}$, is equal to $k(\mathrm{EAB})=k^{\prime}(\mathrm{ECD}) . *$ For alternative addition sequences, and at high substrate concentrations eqn. (2) becomes $\mathrm{E}_{t}=(\mathrm{ECD})\left(1+k / k^{\prime}\right)$. Thus it follows that $\mathrm{R}^{\prime \prime}=\mathrm{E}_{t} k k^{\prime} /\left(k+k^{\prime}\right)$. From this, and eqn. (8), the ratio of the equilibrium reaction rate to the rate of chemical transformation would be:

$$
\mathrm{R} / \mathrm{R}^{\prime \prime}=1 /\left(1+k^{\prime} / k_{-7}+k / k_{-4}\right)
$$

When $\left.\left.k_{-7}\right\rangle\right\rangle k^{\prime}$, and $\left.\left.k_{-4}\right\rangle\right\rangle k, \mathrm{R}$ will $=\mathrm{R}^{\prime \prime}$. Similarly, when $\left.\left.k_{-5}\right\rangle\right\rangle k^{\prime}$ and $k_{-2}$ ) $k, \mathrm{R}^{\prime}$ will $=\mathrm{R}^{\prime \prime}$ at high substrate concentrations. These are the same conditions as for ternary complex interconversion being rate limiting with alternative orders of substrate addition. Thus under these circumstances, the equilibrium interconversion rate would equal the chemical transformation rate.

\section{DISCUSSION}

The relationships developed in this paper for an enzyme system as described by eqn. (1) show that measurement of equilibrium rates may be a powerful tool for detection of compulsory pathways of substrate binding, for discerning whether or not ternary complex interconversion is rate-limiting, for finding mini-

* The rate of actual chemical transformation in any enzymic reaction whether or not at equilibrium must equal but may far exceed the rate of net reaction or the highest equilibrium interconversion rate of any substrate pair or atoms of substrates. Rapid chemical transformation of enzyme-bound reactants accounts for a rapid transfer of oxygen from the glutamate carboxyl to the terminal phosphoryl group of ATP without formation of free orthophosphate ${ }^{3}$. 
mal values of dissociation constants, and for gaining information about relative velocities of certain steps. These considerations have been applied to results from liver and yeast alcohol dehydrogenases and to skeletal and heart muscle lactate dehydrogenase as presented elsewhere ${ }^{4}$. With liver alcohol dehydrogenase alternative order addition of substrates is found, with dissociation of coenzymes as rate-limiting steps. This is in agreement with the extensive studies of Theorell and associates ${ }^{\theta}$ but not with recent conclusions of Dalziel ${ }^{10}$. A similar situation holds with yeast alcohol dehydrogenase, in contrast to the conclusions of Nygaard and Theorell ${ }^{11}$ and of. Mahler and Douglas ${ }^{12}$. Compulsory order of binding is found with lactate dehydrogenase, particularly with bovine heart lactate dehydrogenase at $\mathrm{pH}$ 7.9. These results are in harmony with data of Takenaka and Schwert ${ }^{13}$. With hexokinase, in agreement with Zewe and Fromm ${ }^{14}$ but not with Hammes and Kochavi ${ }^{15}$, an alternative order of substrate addition is found. In addition, particularly with the alcohol dehydrogenase, inhibitory effects on alcohol and acetaldehyde interconversions and information about the chemical transformation steps were obtainable.

The equations as given are based upon rates of interconversion of substrates at equilibrium, and are exact for the systems described. Isotopes provide the only known method for measurement of such rates; in their use certain assumptions must be made, as well as appropriate corrections for approach to isotope equilibrium used in evaluation of rates ${ }^{1}$.

Equations are presented in this paper only for interconversion of $A$ with $C$ and of $B$ with D. Appropriate relationships may, of course, be readily derived for other interconversions such as $A$ with $B$ or $A$ with $D$, as well as for a wide variety of other enzyme reaction schemes. Such extensions can perhaps best be done in connection with appropriate experimental data.

Methodology limitations and restriction of the equilibrium set some obvious limits to the applications of equilibrium rate measurements, but the potentialities, as well as the results obtained to date, would appear to warrant much broader application.

\section{REFERENCES}

1. Boyer, P. D. Arch. Biochem. Biophys. 82 (1959) 387.

2. Boyer, P. D. and Graves, D. J. Proc. Symp. IV, 4th Intern. Congr. Biochem., Moscow, August 1961. Pergamon Press. In press.

3. Graves, D. J. and Boyer, P. D. Biochem. 1 (1962) 739.

4. Silverstein, E. Ph. D. Thesis, Univ. of Minnesota, 1963.

5. Morales, M. F., Horowitz, M. and Botts, J. Arch. Biochem. Biophys. 99 (1962) 258.

6. Alberty, R. A., Bloomfield, V., Peller, L. and King, E. L. J. Am. Chem. Soc. 84 (1962) 4381 .

7. Cleland, W. W. Biochim. Biophys. Acta 67 (1963) 104.

8. Boyer, P. D., Mills, R. C. and Fromm, H. J. Arch. Biochem. Biophys. 81 (1959) 242.

9. See Sund, H. and Theorell, H. In Boyer, P. D., Lardy, H. A. and Myrbäck, K. The Enzymes, 2nd Ed., Academic Press, N. Y., 1963, Vol. VII, p. 25.

10. Dalziel, K. Biochem. J. 84 (1962) 244; Nature 197 (1963) 462.

11. Nygaard, A. P. and Theorell, H. Acta Chem. Scand. 9 (1955) 1300, 1551.

12. Mahler, H. R. and Douglas, J. J. Am. Chem. Soc. 79 (1957) 1159.

13. Takenaka, Y. and Schwert, G. W. J. Biol. Chem. 223 (1956) 157.

14. Zewe, V. and Fromm, H. J. J. Biol. Chem. 237 (1962) 1668.

15. Hammes, G. G. and Kochavi, D. J. Am. Chem. Soc. 84 (1962) 2069, 2073, 2076.

Received April 1, 1963. 